\title{
Erratum to: Effects of a healthy Nordic diet on plasma 25-hydroxyvitamin D concentration in subjects with metabolic syndrome: a randomized, controlled trial (SYSDIET)
}

\author{
Lea Brader • Lars Rejnmark • Carsten Carlberg • Ursula Schwab • \\ Marjukka Kolehmainen · Fredrik Rosqvist • Lieselotte Cloetens • \\ Mona Landin-Olsson • Ingibjorg Gunnarsdottir - Kaisa S. Poutanen • \\ Karl-Heinz Herzig • Ulf Risérus · Markku J. Savolainen · Inga Thorsdottir • \\ Matti Uusitupa $\cdot$ Kjeld Hermansen
}

Published online: 30 March 2014

(c) Springer-Verlag Berlin Heidelberg 2014

\section{Erratum to: Eur J Nutr}

$$
\text { DOI 10.1007/s00394-014-0674-3 }
$$

The article title contains an error in the original publication. The correct title should read as follows:

Effects of a healthy Nordic diet on plasma 25-hydroxyvitamin D concentration in subjects with metabolic syndrome: a randomized, controlled trial (SYSDIET)

The online version of the original article can be found under doi:10.1007/s00394-014-0674-3.

L. Brader $(\bowtie) \cdot$ L. Rejnmark $\cdot$ K. Hermansen

Department of Endocrinology and Internal Medicine, Aarhus University Hospital, Tage-Hansens Gade 2, 8000 Århus C,

Denmark

e-mail: brader.lea@gmail.com

C. Carlberg

Institute of Biomedicine, University of Eastern Finland, Kuopio, Finland

U. Schwab · M. Kolehmainen - K. S. Poutanen - M. Uusitupa Institute of Public Health and Clinical Nutrition, University of Eastern Finland, Kuopio, Finland

F. Rosqvist · U. Risérus

Department of Public Health and Caring Sciences, Clinical

Nutrition and Metabolism, Uppsala University, Uppsala, Sweden

\section{Cloetens}

Biomedical Nutrition, Pure and Applied Biochemistry,

Lund University, Lund, Sweden

\section{Landin-Olsson}

Department of Endocrinology, Skåne University Hospital, Lund, Sweden
I. Gunnarsdottir - I. Thorsdottir

Unit for Nutrition Research, Landspitali - The National

University of Iceland, Faculty of Food Science and Nutrition, School of Health Sciences, University of Iceland, Reykjavík, Iceland

K.-H. Herzig

Department of Physiology and Biocenter of Oulu, Institute of Biomedicine, Oulu University, Oulu, Finland

K.-H. Herzig

Medical Research Centre Oulu, Oulu University Hospital, Oulu, Finland

M. J. Savolainen

Department of Internal Medicine and Biocenter, Institute of Clinical Medicine, University of Oulu, Oulu, Finland

M. J. Savolainen

Clinical Research Centre, Oulu University Hospital, Oulu, Finland 